See discussions, stats, and author profiles for this publication at: https://www.researchgate.net/publication/356514730

\title{
EXPRESS: Age-related differences when searching in a real environment: The use of semantic contextual guidance and incidental object encoding
}

Article in Quarterly Journal of Experimental Psychology (2006) · November 2021

DOI: $10.1177 / 17470218211064887$

CITATIONS

0

3 authors:

Q

Hanane Ramzaoui

Université de Paris

9 PUBLICATIONS 9 CITATIONS

SEE PROFILE

Sara Spotorno

Keele University

33 PUBLICATIONS 140 CITATIONS

SEE PROFILE

Some of the authors of this publication are also working on these related projects:

Interplay between semantic and emotional informations during visual scene perception View project

Project Perception/Action View project
READS

16

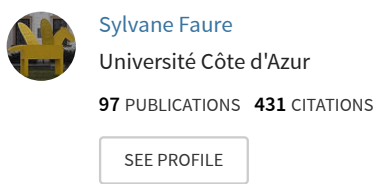


Age-related differences when searching in a real environment: The use of semantic contextual guidance and incidental object encoding

Hanane Ramzaoui ${ }^{1}$, Sylvane Faure ${ }^{1} \&$ Sara Spotorno $^{2}$

Université Côte d'Azur, LAPCOS, France ${ }^{1}$

School of Psychology, Keele University, United Kingdom²

\section{Author Note}

Hanane Ramzaoui was supported by the doctoral studentship award of the Médéric Alzheimer Foundation.

Correspondence should be sent to Hanane Ramzaoui, Laboratoire d'Anthropologie et de Psychologie Cliniques, Cognitives et Sociales (LAPCOS), Université Côte d'Azur, Campus Saint Jean d'Angély / MSHS Sud-Est, 3 Boulevard François Mitterrand, 06357 Nice Cedex 4, France. Tel.: +33 4898814 60. E-mail: hanane.ramzaoui@univ-cotedazur.fr. 


\begin{abstract}
Visual search is a crucial, everyday activity that declines with aging. Here, referring to the environmental support account, we hypothesized that semantic contextual associations between the target and the neighboring objects (e.g., a teacup near a tea bag and a spoon), acting as external cues, may counteract this decline. Moreover, when searching for a target, viewers may encode information about the co-present distractor objects, by simply looking at them. In everyday life, where viewers often search for several targets within the same environment, such distractor objects may often become targets of future searches. Thus, we examined whether incidentally fixating a target during previous trials, when it was a distractor, may also modulate the impact of aging on search performance. We used everyday object arrays on tables in a real room, where healthy young and older adults had to search sequentially for multiple objects across different trials within the same array. We showed that search was quicker: (1) in young than older adults, (2) for targets surrounded by semantically associated objects than unassociated objects, but only in older adults, and (3) for incidentally fixated targets than for targets that were not fixated when they were distractors, with no differences between young and older adults. These results suggest that older viewers use both environmental support based on object semantic associations and object information incidentally encoded to enhance efficiency of real-world search, even in relatively simple environments. This reduces, but does not eliminate, search decline related to aging.
\end{abstract}

Keywords: visual search, aging, real environment, semantic contextual guidance, incidental encoding, aging. 


\section{Introduction}

Visual search is a fundamental task in everyday life, where we often need to locate a target (e.g., our keys) among distractors in cluttered environments. Previous studies conducted in the laboratory, mainly using simple objects arrays, have reported that visual search ability is affected by aging (see for review Madden, 2007). Indeed, older adults are slower than young adults when they have to find a target defined by a conjunction of features such as a specific combination of a shape and colour (e.g., a red square amongst red circles and blue squares; e.g., Foster et al., 1995; Humphrey \& Kramer, 1997; Madden et al., 1996; Whiting et al., 2005). This is interpreted as an impairment in top-down, voluntary attention, which has to be sequentially shifted from item to item to identify the target (but see Wiegand \& Wolfe, 2020), with each shift increasing the total search time. However, studies have reported that older adults perform comparably to young adults when the target pops out in terms of a single feature, for instance, unique colors or orientations alone (e.g., a red circle amongst blue circles), which provides strong bottom-up guidance and can be detected with parallel processing (e.g., Humphrey \& Kramer, 1997; Whiting et al., 2005).

Thus, age-related deficits in visual search can be attributed to inefficiencies of processing due to a decline in (voluntary) attentional resources instead of a decline of the underlying mechanisms. This has been also found in the memory domain (Craik, 1983). Crucially, providing environmental support, also called contextual support, can reduce agerelated differences (Craik \& Byrd, 1982; see also e.g., Dunlosky \& Hertzog, 1998; Froger et al., 2012; Taconnat et al., 2009; Woodbridge et al., 2018). It involves presenting external cues at encoding and/or retrieval, for example, semantic cues (like the name of the category 'insect' for the word 'ant'). These cues can promote effective strategies to process information in more depth, especially in older adults (e.g., Craik, 1986; Craik \& Byrd, 1982; Shaw \& Craik, 1989; Smith et al., 1998) and, therefore, facilitate retrieval processes (Craik, 
1986; Craik \& McDowd, 1987). Beyond the memory domain, older adults rely more on information provided by the environment than young adults in other domains, such as perception or action management (see Lindenberger \& Mayr, 2014).

One form of environmental support for visual search can be provided by spatial cues. For instance, the location of the target with respect to that of the other simultaneously present items (i.e., using repeated item configurations) can be invariant across searches. Both young and older adults can use this location predictability to shorten search times, as it has been shown in the laboratory mainly using simple arrays, for instance composed by letters s (e.g., Howard et al., 2004; Preuschhof et al., 2019; but see also Smyth \& Shanks, 2011).

Guidance by context during search: The use of semantic contextual cues

Besides the ad-hoc situations set up in the laboratory to create and manipulate predictability, regularities are an important part of our everyday life, on which we base predictions about the world to guide behaviour. Everyday visual environments contain not only spatial but also semantic regularities that allow us to predict, for instance, whether and where we will find certain objects. These regularities can act as powerful sources of environmental support and are learned through lifetime experience, when individuals store information about the different environments/scene contexts in long-term memory representations (i.e., schemas; see Biederman et al., 1982) that include the expected (i.e., semantically consistent) objects in a specific scene and their expected locations (e.g., Henderson, 2003). For instance, it would be surprising to find a pan in a bathroom, or to find it on the floor in a kitchen.

Research on guidance by context has been extensively conducted on young adults using static real-world scene images (pictures of everyday environments, like kitchens or streets) presented on a lab's computer screen, and often manipulating object-scene semantic 
consistency. A number of studies have shown that target objects that are semantically consistent with the scene are found sooner when searching than semantically inconsistent objects (e.g., Castelhano \& Heaven, 2011; Henderson et al., 1999; Võ \& Henderson, 2009). In the literature on cognitive aging, the few studies using real-world or 3D pseudo-realistic scenes have suggested that, compared to young adults, older adults rely more on expectations about object-scene semantic and spatial consistency (Borges et al., 2020; Neider \& Kramer, 2011). Indeed, Borges et al. (2020) found longer search times in older than in young adults for inconsistent objects included in scenes (e.g., an iron in a restaurant) that were primed with another scene image from the same category (e.g., a different restaurant). Neider and Kramer (2011) found that older adults benefited more than young adults from contextual guidance related to searching for a semantically and spatially consistent object (e.g., a jeep on the ground) compared to searching for a fictional object that could not elicit any expectations (an "oleh", appearing either on the ground or in the air).

To investigate the influence of context on visual search guidance, research on young adults has also manipulated object co-occurrence, which concerns objects' semantic association and spatial proximity in everyday contexts (for instance, cutlery, plates and glasses tend to occur together in kitchens and to be placed relatively closed one another; e.g., Bar, 2004; Biederman \& Gerhardstein, 1995; Henderson et al., 1987). Knowledge about the presence of specific objects that usually appear with the target within a certain scene provides information that guides eye movements and facilitates search (e.g., Hwang et al., 2009; Koehler \& Eckstein, 2017; Mack \& Eckstein, 2011). In a real environment, and again including only young adults, Mack and Eckstein (2011) studied how contextual cues provided by object co-occurrence influence search for common target objects placed on a table in a real room. Search was quicker when the target object was presented next to a cue object with which it usually co-occurs in everyday contexts (such as a fork next to a plate) than 
when it was surrounded by unrelated, not usually co-occurring objects. In addition, viewers made more fixations on cue objects related to the target than on those unrelated. These findings demonstrated that semantic contextual cueing provided by object co-occurrence guides eye movements and improves search efficiency in real environments.

From the literature reviewed here, it clearly emerges that our understanding of how older adults search for objects comes from studies in the laboratory, which have typically used arrays of simple items and, in fewer occasions, real-world scene images. To the best of our knowledge, no study has investigated search performance in older adults within a real environment. In everyday life, activity-specific aims may change rapidly while the environment remains relatively constant, and visual search often requires looking for different objects in the same, familiar environment. For instance, we may search for our car keys, wallet and phone in the same room and, often, even on the same table. By contrast, in the laboratory, studies on visual search typically present a new search display for each trial (e.g., Castelhano \& Heaven, 2011; Henderson et al., 1999; Spotorno et al., 2015; Võ \& Henderson, 2009). This introduces a fundamental difference from the repeated searches within a given environment, where the viewer has greater opportunities to incidentally encode information about the location and the identity of the co-occurring objects.

\section{The role of incidental encoding in visual search}

Previous research, mainly conducted on young adults, has examined how memory for objects is incidentally acquired during search. In particular, several studies have investigated the role of eye movements in building memory representations during search. Viewing involves a succession of eye fixations during which visual information is gathered. It has been shown that visual information accumulates across fixations (e.g., Pertzov et al., 2009; Tatler et al., 2005). This happens not only during intentional memorisation. Objects are 
encoded into memory incidentally during search, both when they are the searched targets (e.g., Draschkow et al., 2014; Hout \& Goldinger, 2012; Josephs et al., 2016; Williams et al., 2009) and when they are search distractors (Hout \& Goldinger, 2012; Williams, 2010; Williams et al., 2005, 2009). Moreover, it is well established that search becomes much quicker when the same target object is repeatedly searched for and, therefore, repeatedly fixated (e.g., Võ \& Wolfe, 2012). Indeed, while search performance greatly improves when the same target object is repeatedly searched for (e.g., Võ \& Wolfe, 2012), mixed findings have been reported in the laboratory when searching for objects that were fixated in previous searches when they were not the target within the same scene image (Hollingworth, 2012; Võ \& Wolfe, 2012; Wolfe et al., 2011) or within object arrays that maintain some object identity and location information across trials (Hout \& Goldinger, 2010, 2012), showing either shorter search times or no differences compared to search of novel objects. Findings have shown either shorter search times (Hout \& Goldinger, 2010, 2012) or no differences (Võ \& Wolfe, 2012; Wolfe et al., 2011) for these objects compared to search of novel objects. To our knowledge, the only investigation of repeated search in a real environment (Howard et al., 2011), where individuals searched for two targets within the same set of objects placed on a table in two subsequent trials, did find faster search when the target had been incidentally fixated in the previous trial (when it was a distractor) than when it had not been fixated. This result provides support to the idea that task relevance may not be essential to form object memory representations from object fixation that can be subsequently used during visual search.

All the work here examined only included young adults. The only study that, to our knowledge, examined age-related differences in incidental memory for targets and distractors formed during visual search (Williams et al., 2009) used different arrays of everyday objects presented on a screen, with a different target in each array among related (same category or 
same color as the target) and unrelated distractors. Search was followed by a surprise two alternative-forced-choice recognition test. During search, older adults fixated all objects for longer than young adults, and particularly so the target object. These longer viewing durations did not result in better memory: Recognition was similar in older and young adults for all types of distractors, and was worse in older adults for targets. This might suggest that memory representations from object fixation are formed less efficiently with aging. However, regressing total fixation time and number of viewings on memory performance, the authors found that object memory improved similarly in both young and older adults the more the object were viewed. The only exception was a greater memory benefit (larger slope) in young compared to older adults across multiple object viewings only for color-related distractors. The poorer memory for target in older adults seemed to depend on lower intercept, not information accumulation rates. One possible explanation for this may concern information processing levels (Craik \& Lockhart, 1972): Targets would be processed more in depth than distractors, and would thus require a greater involvement of top-down mechanisms, which become less effective with age (Craik \& Byrd, 1982). However, worse incidental memory in older than young adults has also been reported when the encoding task does not distinguish between task-relevant and irrelevant objects: For instance, in Shih et al.'s (2012), where free viewing of an image of a room with several objects was followed by a surprise identity (old/new recognition) and position (relocation) memory test, both performed with real objects, in the real room depicted in the image. In this study, no age-related differences were found in how information for object identity and position accumulated during and across fixations, with comparable memory benefits in young and older adults when an object was viewed more frequently or for longer. 
The present study

Age-related differences in visual search have previously only been inferred from laboratory studies. However, natural behaviour may considerably differ from viewing static, often oversimplified images on a computer screen. By presenting conditions far removed from those of the real world, laboratory studies may have limited validity in investigating the complex interactions between the allocation of attention in the environment and memory representations of the targets and their surroundings that characterise everyday search behaviour (see Kingstone et al., 2008). This study aimed to reduce this limitation by investigating age-related search differences in a real-world environment, while maintaining a degree of control over the surroundings that allows experimental manipulation of the relationships between targets and distractor objects. To this purpose, we recorded eye movements using a mobile eye-tracker while both young and older adults had to search for different everyday, real objects placed on real tables in a room. More precisely, we examined the impact of healthy aging on the use of two sources of guidance typically present in everyday search: (1) expectations arising from semantic knowledge about object cooccurrence (association) in a given context, and (2) target object memory representations formed via incidental encoding when the object was looked at in a previous trial even though it was a distractor.

We used a paradigm similar to that of Mack and Eckstein's (2011) study, in which participants had to look sequentially for several target objects placed on the same table and the semantic context was manipulated by varying the spatial proximity between target objects and semantically associated objects. We hypothesized that, while older adults should overall search more slowly than younger adults, this performance gap should be reduced when the target is surrounded by semantically associated distractors compared to when it is surrounded by unassociated distractors. Indeed, older adults should rely more than young adults on 
Author Accepted Manuscript pase oofzz

contextual expectations (e.g., Borges et al., 2020; Neider \& Kramer, 2011) and, thus, they should benefit more from the environmental support (Craik \& Byrd, 1982) provided by object associations acting as semantic cues.

To study the impact of aging on the use of incidental information gathered during search, we examined the search times in young versus older adults for targets that were fixated in previous trials (when they were distractors) compared to the search times for targets that were not previously fixated. We remained exploratory, aiming to investigate whether and how such impact may emerge when searching in a real environment. The rare studies on agerelated differences in incidental object memory had used different paradigms and reported mixed evidence, with either no age-related differences in distractor incidental memory following a visual search lab-based task (Williams et al., 2009) or worse incidental memory in older than young participants following free viewing (Shih et al., 2012). Moreover, they had shown either little (Williams et al., 2009) or no (Shih et al., 2012) reduction in information accumulation during object fixation with aging. Thus, we deemed that these previous findings were insufficient to expect either no age-related differences or a reduced search benefit of incidental object encoding in our study.

\section{Method}

\section{Participants}

Sixty-four volunteers, 32 older adults and 32 young adults, participated in the study (Age: $M=68.41$ and $S D=3.43$ in older adults, and $M=21.66$ and $S D=1.62$ in young adults; gender: Ten males and 22 females in each age group). Older adults were volunteers recruited in the wider community. Young adults were psychology university students. The two age groups did not differ in education levels (older adults: $M=14.44$ years, $S D=$ 2.79; young adults: $M=14.78$ years, $S D=1.98$ ). Participants had no history of head 
trauma and no ophthalmologic impairments (e.g., cataract, macular degeneration, or glaucoma). Older participants showed a Mini Mental State Examination score (Folstein et al., 1975; Kalafat et al., 2003) of a minimum of $28(M=29.06, S D=.98)$. Their verbal episodic memory was assessed by the Free and Cued Selective Reminding Test (Grober et al., 1988) $(M=47.17, S D=1.38)$. Older participants also performed the 80 items Picture Naming task from Deloche and Hannequin (1997) to ensure that they did not have any deficit in visual object recognition $(M=79.72, S D=.67)$. The study was conducted in accordance with the Declaration of Helsinki. Ethical approval was obtained from the Protection of Persons Committee “Sud Méditerranée V” (CPP N 16036, July 13th, 2016). Informed written consent was obtained from all participants.

\section{Apparatus}

Eye movements were recorded with eye-tracking glasses (Eye Tracking Glasses 2, SensoMotoric Instruments: SMI), sampling at $60 \mathrm{hz}$. The eye-tracking glasses were worn as a normal pair of glasses. They included three cameras: Two cameras recording infrared corneal reflection for binocular eye tracking and a scene camera. Data were collected on a customized Samsung android device, which was connected to the eye tracking glasses. Gaze position accuracy is of $0.5^{\circ}$ over all distances. Search times and fixations were extracted offline from video recordings of the eye tracker (see Data Analysis).

Calibration was performed using a three-point procedure to ensure that data accurately reflected the position of the gaze on the table. The participant stood at approx. $60 \mathrm{~cm}$ from a table (the same distance as for performing the search task) while looking as accurately as possible at a colored square without moving the head. Each of the three colored squares was indicated by the experimenter's pointing with the index finger. Snap-on corrective lenses $(+/-$ 4 diopter, varying in 0.50 ) were used for participant whose visual acuity needs to be corrected to normal. 


\section{Materials}

The search task used object arrays on four tables in a real room, four targets and 14 distractors per table (Figure 1). Each table contained four semantic groups formed by four associated objects, which usually co-occur in everyday life. The objects pertaining to two semantic groups were placed near each other, while the objects of the other two groups were placed far apart from each other. Moreover, there were two additional distractor objects, unrelated to all other objects, on each table (see Table $\mathbf{1}$ in the online Supplementary Materials, for details of objects in each search table).

\section{--- Insert Figure 1 about here ---}

Four search targets (here named S1/S2/S3/S4 according to the order they were looked for) were designated for each table (Figure 2). Two targets belonged to one semantic group and were surrounded by the semantically associated objects (near target condition), and two targets belonged to the other semantic group and were located far from the semantically associated objects (far target condition).

\section{--- Insert Figure 2 about here ---}

\section{Procedure}

Participants performed the task individually and were instructed to search for four targets, one after the other, on each of the four tables (16 trials), as quickly and as accurately as possible. On entering the room, all tables were covered by a paper tablecloth to ensure that participants looked at the table arrays only from the very beginning of the trial. First, participants stood in front of the table (on a floor marking) at a distance of about $60 \mathrm{~cm}$ from the centre of the table. At the beginning of each trial, they had to stare at the wall in front of them. For each table, the experimenter called out the name of the first target and then 
removed the paper tablecloth. After this, the participant had to repeat the target name, to ensure that they heard it well, before starting the search. Then, the participant was allowed to move their eyes and head freely while looking at the table arrays to search for the given target. The participant was asked to point the target with the index finger once located, as soon as possible, and to notify it orally (by saying "I found it!"). This procedure was used to confirm whether or not the participant had correctly identified the target. At the end of the trial, the participant had to stare at the wall again until the experimenter called out the name of the next target.

The trials started (S1) with a near target in half of the tables and then alternated between the two conditions (i.e., searched target order: S1/near, S2/far, S3/near, S4/far for two tables, and vice-versa for the other two tables). For each table, the object position and the searched target order was the same for all participants, but the order of the tables was counterbalanced across participants according to a Latin square.

\section{Results}

\section{Data analysis}

Using Begaze Analyses Software (SMI), search times and the presence of incidental fixations were hand annotated on a frame-by-frame basis from the video recording of the eye tracker. The video recording from the scene camera includes the gaze cursor that indicates the location at which the participant is gazing across the scene view. Response time during search was defined as the time elapsed (in milliseconds) between the frame of the first fixation on the search table and the first frame in which the participant pointed the target. For each target object, fixations on that object in the previous trials when it was a distractor (henceforth called "incidental fixations") were annotated as 1 in the presence of fixation(s) and 0 in the absence of fixation. Any point of gaze (reflected by the gaze cursor) on a target object in the 
Author Accepted Manuscript page 14 of 32

trials when it was a distractor, which lasted at least one frame, was considered as presence of incidental fixations on that object. Please note that incidental fixations could only been made on S2 to S4 targets.

We excluded from analyses trials with errors (70 trials): Responses were coded as correct if the participant looked at the target while pointing to it. Trials with search times greater than 2.5 standard deviations from the mean (17 of the remaining trials for older adults and 11 of the remaining trials for young adults) were excluded as outliers. We then removed trials with search times shorter than $500 \mathrm{~ms}$ (seven of the remaining trials for older adults and three of the remaining trials for young adults), considered as reflecting anticipatory responses. As a result, of the 1024 possible trials (64 participants $\mathrm{x} 16$ trials each), 916 trials were included in the final analysis $(89.45 \%)$. Incidental fixations were made in 252 included trials (27.51\%). All participants made incidental fixations. All but two participants (one young, one older) made incidental fixations in the near target condition and all but six participants (three young, three older) made incidental fixations in the far target condition. The analyses described below included all participants; however, we also conducted the same analyses removing the participants without incidental fixations in either the near or far condition, and we found the same patterns of results.

Statistical analyses were performed using R version 3.3.1 (The R Foundation of Statistical Computing). We carried out Linear Mixed Models (LMMs) using the lmer function from the lme4 package (Bates et al., 2015), and entering each trial as a separate data point. LMMs allow simultaneous estimation of between-subject and between-item variance (Klieg1 et al., 2011), and a better preservation of statistical power in the presence of data removal (Baayen, 2008). We analyzed search time, with Age Group (young vs. older), Target Position (near vs. far from semantically associated objects), Incidental Fixation (presence of incidental fixations - i.e., the target was fixated when it was a distractor vs. no 
incidental fixation), Trial Order (1 to 16) as interactive predictors. Trial order was included to disentangle the potential search facilitation due to incidental fixations from the general search facilitation that may simply be due to familiarisation with the task. Participants and items (target objects) were specified as random factors. As the maximal model including all the possible crossed random effects did not converge, we simplified it in a stepwise fashion, starting by removing the correlations between the random slopes and the intercepts. Then, we removed the slope of the highest order interaction between the predictors, and we gradually reduced the model until it converged. We simplified the item term before the subject term. The most complex random effect structure of the model that converged and was not singular included previous fixation and target position slopes for the participant term, and the age group and trial order slopes for the item term. No slopes of interactions were included. There were no other models with random effect structures of equal complexity that converged and were not singular. The categorical predictors were recoded using sum (deviation) coding, which compares the mean of the dependent variable for a given level of the overall mean of the dependent variable over all the levels. Search times were logtransformed to meet the model assumptions of normally distributed data. We report the predictors' coefficients $(\beta)$ values and standardized coefficients $\left(\beta \_s t d\right)$ values, the $S E$ values, the $t$ values of the predictors, and the associate $p$ values. $\beta \_$std generated using sjstats library (Lüdecke, 2019) and $p$ values generated using the lmerTest library (Kuznetsova et al., 2017). Follow-up LMMs were carried out to analyse simple effects in the case of significant interactions $(p<.05)$. Supplementary Materials describe the structure of the models used. Graphics were created using the ggplot2 package (Wickham, 2009). The observed and the fitted values (i.e., the predicted values from the dataset used in model fitting) from the LMM model were plotted.

\section{Search performance}




\section{Author Accepted Manuscript page 16 of 32}

In Figure 3, search time (plots 1A and 2A) and fitted values (plots 1B and 2B) are shown as a function of target position (near vs. far from semantically associated objects) and age group, and as a function of incidental fixation on the target (the presence of incidental fixations vs. no incidental fixation) and age group. There was a main effect of the age group, $\beta=.290, S E=.066, t=4.39, p<.001, \beta \_s t d=.372$, with search being faster for young adults $(M=1064 \mathrm{~ms}, S D=393 \mathrm{~ms})$ than for older adults $(M=1487 \mathrm{~ms}, S D=704 \mathrm{~ms})$, and a main effect of incidental fixation, $\beta=.164, S E=.068, t=2.41, p=.02, \beta \_s t d=.129$, as search was faster following the presence of incidental fixations $(M=1199 \mathrm{~ms}, S D=532 \mathrm{~ms})$ compared to no incidental fixation $(M=1300 \mathrm{~ms}, S D=629 \mathrm{~ms})$. There was no significant main effect of trial order, $\beta=-.002, S E=.003, t<1, p=.520$, nor of target position, $\beta=.099, S E=.095$, $t=1.04, p=.309, \beta \_s t d=.123$. However, we found an interaction between the age group and the target position, $\beta=.242, S E=.110, t=2.03, p=.045, \beta \_s t d=.100$. Search for targets surrounded by semantically associated objects was faster for young adults $(M=1030 \mathrm{~ms}, S D$ $=360 \mathrm{~ms})$ than for older adults $(M=1335 \mathrm{~ms}, S D=592 \mathrm{~ms}), \beta=.237, S E=.058, t=4.11, p$ $<.001, \beta \_s t d=.551$ (follow-up Model A). Search for targets located far from semantically associated objects was also faster for young adults $(M=1100 \mathrm{~ms}, S D=422 \mathrm{~ms})$ than for older adults $(M=1662 \mathrm{~ms}, S D=779 \mathrm{~ms}), \beta=.406, S E=.059, t=6.91, p<.001, \beta \_s t d$ $=.945$ (follow-up Model A). The effect of group was greater for targets located far from semantically associated objects than for those surrounded by semantically associated objects. While no significant differences were found depending on the target position in young adults, $\beta=.046, S E=.091, t<1, p=.621, \beta \_s t d=.104$, search in older adults was faster for targets surrounded by semantically associated objects $(M=1335 \mathrm{~ms}, S D=592 \mathrm{~ms})$ than for those located far from them $(M=1662 \mathrm{~ms}, S D=779 \mathrm{~ms}), \beta=.214, S E=.091, t=2.35, p=.030$, $\beta \_s t d=.497$ (follow-up Model B). We found no other significant interaction, all $t s \leq 1.34$, all $p s \geq .182$. 


\section{Discussion}

The present study examined whether and how aging modulates eye-movement guidance provided by semantic knowledge and by incidentally generated memory representations formed during search for real objects in a real environment. To this purpose, we analyzed the influence of semantic association (relatedness in terms of likelihood of cooccurrence in everyday life) between each target and the neighboring objects, and the potential benefit of incidental encoding of information about the target object in previous trials, when it was a distractor.

We hypothesized that search would be overall slower in older adults, but also that semantic guidance provided by the co-present objects would improve performance in both young and older adults, with a greater influence in older adults. We reported that only older adults found the target more quickly when it was surrounded by semantically associated objects rather than unassociated objects. This shows that older adults used expectation arising from learned object associations to direct search efficiently to the locations most likely to contain the target. This result extends to the real world previous laboratory research showing that reliance on semantics during search increases with aging (Borges et al., 2020). Moreover, showing that semantic cues reduce age-related differences in performance, our study corroborates the importance to provide external aid to reduce the impact of cognitive aging on memory, coherently with the hypothesis of environmental support (Craik \& Byrd, 1982).

The absence of an effect of object semantic association on search guidance in our group of young participants is contrary to Mack and Eckstein's (2011) findings. This may depend on differences between the materials and procedures of the two studies: First, participants were closer to the table in our experiment than in Mack and Eckstein's study 
Author Accepted Manuscript page 18 of 32

(approx. 24 versus 60 to 96 inches, respectively); second, our object arrays were less cluttered than theirs. Both aspects may have made search easier in our study and, consequently, may have reduced the reliance on semantic cues in our young participants.

We expected that incidental information gathering through fixation would guide search, but concerning the impact of aging on incidental encoding our study had to remain exploratory, with some scarce previous research (Shih et al., 2012; Williams et al., 2009) suggesting either no differences between older and young adults or a somewhat smaller memory benefit from object fixation in older adults. We found that, regardless of the age group, search was quicker for targets that had been fixated when they were distractors in preceding trials. This suggests that incidental information gathering from an object led to a memory representation binding identity and position, which was sufficiently robust and detailed in older adults to facilitate search as in young adults. Our findings, therefore, extend to older adults the search benefit from incidental encoding of object information that Howard et al. (2011) found in young adults. It is also important to note that we found no interaction between the order of the trial and the search benefit due to incidental fixation, suggesting that our results dissociate the search facilitation due to incidental fixation from the general search facilitation due to task familiarisation.

Overall, our study suggested that incidentally encoded memories were a more prominent facilitator of search than semantic contextual guidance in young adults, as they benefited from previously fixating a target, but not from the semantic associations between targets and surrounding distractors. Older adults, instead, benefited from both incidentally generated target object memory and semantic contextual guidance provided by the targetdistractors co-occurrence. This is in line with studies highlighting that, with advanced age, the use of cues in the environment as a source of "external memory" (e.g., making shopping lists, writing down appointments on a calendar) increases (Bouazzaoui et al., 2010; Lindenberger 
\& Mayr, 2014). Therefore, we may conclude that, at least in relatively simple real environments as the one used in this study, incidentally encoded, memory representations of the specific target object seem to be enough to guide search effectively in young adults. However, in the same, simple environments, older adults may employ more strategies to enhance their search performance, in particular by also relying on semantic cues provided by the neighboring objects. The use of this supplementary, environmental support (e.g., Craik \& Byrd, 1982) by older adults might be interpreted as a mechanism that compensates for slower information processing speed (Salthouse, 1996), slower eye movements (e.g., Warren et al., 2013), and declining bottom-up, perceptual processing (e.g., Madden et al., 2017), all of which are related to aging. However, in our study, while older adults benefited from semantic contextual guidance and from incidental encoding of objects, this was not enough to bring their search performance up to the level of young adults. Our results highlight the need for future work on visual search in aging to take greater account of the role of guidance by both incidental encoding and semantic cues. By recommending the use of semantic cues to facilitate search, they may also contribute to environmental design in order to support older people, especially those with limited efficiency, in their daily activities, for which an initial and essential component is finding appropriate objects to use.

\section{Supplementary Material}

The Supplementary Material is available at: qjep.sagepub.com

\section{Data availability statement}

The data that support the findings of this study are available from the corresponding author upon request. 


\section{Acknowledgements}

We are very grateful to Léa Bacchi and Emmanuelle Delmasse-Simoni for their assistance in data collection. We are also grateful to Dr Joseph Brooks and Professor Ben Tatler for their comments on a draft of the manuscript.

\section{Authors' contributions}

Hanane Ramzaoui: Conceptualization; Methodology; Formal analysis; Investigation; Data curation; Writing - Original draft; Writing - Review \& Editing; Visualization; Project administration; Supervision. Sylvane Faure: Writing - Review \& Editing; Supervision. Sara Spotorno: Methodology; Formal analysis; Writing - Review \& Editing; Supervision.

\section{Declaration of Conflicting Interests}

The Authors declare that there is no conflict of interest. 


\section{References}

Baayen, R. H. (2008). Analyzing Linguistic Data: A practical introduction to statistics. Cambridge University Press, Cambridge.

Bar, M. (2004). Visual objects in context. Nature Reviews Neuroscience, 5(8), 617-629. doi.org/10.1038/nrn1476

Bates, D., Mächler, M., Bolker, B., \& Walker, S. (2015). Fitting Linear Mixed-Effects Models Using lme4. Journal of Statistical Software, 67(1), 1-48. doi.org/10.18637/jss.v067.i01

Biederman, I., \& Gerhardstein, P. C. (1995). Viewpoint-dependent mechanisms in visual object recognition: Reply to Tarr and Bülthoff (1995). Journal of Experimental Psychology: Human Perception and Performance, 21(6), 1506-1514. doi.org/10.1037/00961523.21.6.1506

Biederman, I., Mezzanotte, R. J., \& Rabinowitz, J. C. (1982). Scene perception: Detecting and judging objects undergoing relational violations. Cognitive Psychology, 14(2), 143-177. doi.org/10.1016/0010-0285(82)90007-X

Borges, M. T., Fernandes, E. G., \& Coco, M. I. (2020). Age-related differences during visual search: the role of contextual expectations and cognitive control mechanisms. Aging, Neuropsychology, and Cognition, 27(4), 489-516.

Bouazzaoui, B., Isingrini, M., Fay, S., Angel, L., Vanneste, S., Clarys, D., \& Taconnat, L. (2010). Aging and self-reported internal and external memory strategy uses: The role of executive functioning. Acta Psychologica, 135(1), 59-66. doi.org/10.1016/j.actpsy.2010.05.007

Castelhano, M., \& Henderson, J. (2005). Incidental visual memory for objects in scenes. Visual Cognition, 12(6), 1017-1040. doi.org/10.1080/13506280444000634

Castelhano, M. S., \& Heaven, C. (2011). Scene context influences without scene gist: Eye movements guided by spatial associations in visual search. Psychonomic Bulletin \& 


\section{Author Acceoted VanuScribt page 22 of 32}

Review, 18(5), 890-896. doi.org/10.3758/s13423-011-0107-8

Craik, F. I. M. (1983). On the transfer of information from temporary to permanent memory [and discussion]. Philosophical Transactions of the Royal Society B: Biological Sciences, 302(1110), 341-359. doi.org/10.1098/rstb.1983.0059

Craik, F. I. M. (1986). A functional account of age differences in memory. In F. K. \& H. Hagendorf (Ed.), Human memory and cognitive capabilities (pp. 409-422). Human memory and cognitive capabilities.

Craik, F. I. M., \& Byrd, M. (1982). Aging and cognitive deficits: The role of attentional ressources. In F. I. M. Craik \& S. Trehub (Ed.), Aging and cognitive processes (pp. 191211). New York: Plenum Press. doi.org/110.1007/978-1-4684-4178-9_11

Craik, F. I. M., \& Lockhart, R. S. (1972). Levels of processing: A framework for memory research. Journal of Verbal Learning and Verbal Behavior, 11(6), 671-684. doi.org/10.1016/S0022-5371(72)80001-X

Craik, F. I. M., \& McDowd, J. M. (1987). Age differences in recall and recognition. Journal of Experimental Psychology: Learning, Memory, and Cognition, 13(3), 474-479. doi.org/10.1037/0278-7393.13.3.474

Deloche, G., \& Hannequin, D. T. (1997). Test de denomination orale d'images DO 80 [Picture Naming Test DO-80]. Paris: Les Editions Du Centre de Psychologie Appliquée.

Draschkow, D., Wolfe, J. M., \& Võ, M. L.-H. (2014). Seek and you shall remember: Scene semantics interact with visual search to build better memories. Journal of Vision, 14(8), 10-10. doi.org/10.1167/14.8.10

Dunlosky, J., \& Hertzog, C. (1998). Aging and deficits in associative memory: What is the role of strategy production? Psychology and Aging, 13(4), 597-607. doi.org/10.1037/08827974.13.4.597

Folstein, M. F., Folstein, S. E., \& McHugh, P. R. (1975). “Mini-mental state”: A practical 
method for grading the cognitive state of patients for the clinician. Journal of Psychiatric Research, 12(3), 189-198. doi.org/10.1016/0022-3956(75)90026-6

Foster, J. K., Behrmann, M., \& Stuss, D. T. (1995). Aging and visual search: Generalized cognitive slowing or selective deficit in attention? Aging, Neuropsychology, and Cognition, 2(4), 279-299. doi.org/10.1080/13825589508256604

Froger, C., Bouazzaoui, B., Isingrini, M., \& Taconnat, L. (2012). Study time allocation deficit of older adults: The role of environmental support at encoding? Psychology and Aging, 27(3), 577-588. doi.org/10.1037/a0026358

Grober, E., Buschke, H., Crystal, H., Bang, S., \& Dresner, R. (1988). Screening for dementia by memory testing. Neurology, 38, 900-903. doi.org/10.1212/WNL.38.6.900

Henderson, J. M. (2003). Human gaze control during real-world scene perception. Trends in cognitive sciences, 7(11), 498-504. doi.org/10.1016/j.tics.2003.09.006

Henderson, J. M., Pollatsek, A., \& Rayner, K. (1987). Effects of foveal priming and extrafoveal preview on object identification. Journal of Experimental Psychology. Human Perception and Performance, 13(3), 449-463. doi.org/10.1037/0096-1523.13.3.449

Henderson, J. M., Weeks, P. A. J., \& Hollingworth, A. (1999). The effects of semantic consistency on eye movements during complex scene viewing. Journal of Experimental Psychology: Human Perception and Performance, 25(1), 210-228. doi.org/10.1037/00961523.25.1.210

Hollingworth, A. (2012). Guidance of visual search by memory and knowledge. In Nebr Symp Motiv (Vol. 59, pp. 63-89). doi.org/10.1007/978-1-4614-4794-8_4

Hout, M. C., \& Goldinger, S. D. (2010). Learning in repeated visual search. Attention, Perception, \& Psychophysics, 72(5), 1267-1282. doi.org/10.3758/APP.72.5.1267

Hout, M. C., \& Goldinger, S. D. (2012). Incidental learning speeds visual search by lowering response thresholds, not by improving efficiency: Evidence from eye movements. Journal 


\section{Author Accepted Manuscript page eat ofz2}

of Experimental Psychology: Human Perception and Performance, 38(1), 90-112. doi.org/10.1037/a0023894

Howard, C. J., Pharaon, R. G., Körner, C., Smith, A. D., \& Gilchrist, I. D. (2011). Visual search in the real world: Evidence for the formation of distractor representations. Perception, 40(10), 1143-1153. doi.org/10.1068/p7088

Howard, J. H., Howard, D. V., Dennis, N. A., Yankovich, H., \& Vaidya, C. J. (2004). Implicit spatial contextual learning in healthy aging. Neuropsychology, 18(1), 124-134. doi.org/10.1037/0894-4105.18.1.124

Humphrey, D. G., \& Kramer, A. F. (1997). Age differences in visual search for feature, conjunction, and triple-conjunction targets. Psychology and Aging, 12(4), 704-717. doi.org/10.1037//0882-7974.12.4.704

Hwang, A. D., Higgins, E. C. E. C., Pomplum, M., \& Pomplun, M. (2009). A model of top-dow attentional control during visual search in complex scenes. Journal of Vision, 9(5), 1-18. doi.org/10.1167/9.5.25.A

Josephs, E. L., Draschkow, D., Wolfe, J. M., \& Võ, M. L. H. (2016). Gist in time: Scene semantics and structure enhance recall of searched objects. Acta Psychologica, 169, 100108. doi.org/10.1016/j.actpsy.2016.05.013

Kalafat, M., Hugonot-Diener, L., \& Poitrenaud, J. (2003). Standardisation et étalonnage français du "Mini Mental State" (MMS) version GRÉCO. Revue de Neuropsychologie (Vol. 13).

Kingstone, A., Smilek, D., \& Eastwood, J. D. (2008). Cognitive ethology: A new approach for studying human cognition. British Journal of Psychology, 99(3), 317-340. doi.org/10.1348/000712607X251243

Kliegl, R., Wei, P., Dambacher, M., Yan, M., \& Zhou, X. (2011). Experimental effects and individual differences in linear mixed models: Estimating the relationship between spatial, 
object, and attraction effects in visual attention. Frontiers in psychology, 1, 238.

Koehler, K., \& Eckstein, M. P. (2017). Beyond scene gist: Objects guide search more than scene background. Journal of Experimental Psychology: Human Perception and Performance, 43(6), 1177-1193. doi.org/10.1037/xhp0000363

Kuznetsova, A., Brockhoff, P. B., \& Christensen, R. H. B. (2017). lmerTest Package: Tests in Linear Mixed Effects Models. Journal of Statistical Software, 82(13). doi.org/10.18637/jss.v082.i13

Lindenberger, U., \& Mayr, U. (2014). Cognitive aging: Is there a dark side to environmental support? Trends in Cognitive Sciences, 18(1), 7-15. doi.org/10.1016/j.tics.2013.10.006

Lüdecke, D. (2019). sjstats: Statistical Functions for Regression Models (Version 0.17.6). doi.org/10.5281/zenodo.1284472

Mack, S. C., \& Eckstein, M. P. (2011). Object co-occurrence serves as a contextual cue to guide and facilitate visual search in a real viewing environment. Journal of Vision, 11(9), 1-16. doi.org/10.1167/11.9.9

Madden, D. J. (2007). Aging and visual attention. Current Directions in Psychological Science, 16(2), 70-74. doi.org/10.1111/j.1467-8721.2007.00478.x

Madden, D. J., Parks, E. L., Tallman, C. W., Boylan, M. A., Hoagey, D. A., Cocjin, S. B., Johnson, M. A., Chou, Y., Potter, G. G., Chen, N., Packard, L. E., Siciliano, R. E., Monge, Z. A., \& Diaz, M. T. (2017). Frontoparietal activation during visual conjunction search: Effects of bottom-up guidance and adult age. Human Brain Mapping, 38(4), 2128-2149. doi.org/10.1002/hbm.23509

Madden, D. J., Pierce, T. W., \& Allen, P. A. (1996). Adult age differences in the use of distractor homogeneity during visual search. Psychology and Aging, 11(3), 454-474. doi.org/10.1037//0882-7974.11.3.454

Neider, M. B., \& Kramer, A. F. (2011). Older adults capitalize on contextual information to 


\section{Author Accepted Manuscript Pageroforz}

guide search. Experimental Aging Research, 37(5), 539-571. doi.org/10.1080/0361073X.2011.619864

Pertzov, Y., Avidan, G., \& Zohary, E. (2009). Accumulation of visual information across multiple fixations. Journal of Vision, 9(10), 2-2. doi.org/10.1167/9.10.2

Preuschhof, C., Sharifian, F., Rosenblum, L., Pohl, T. M., \& Pollmann, S. (2019). Contextual cueing in older adults: Slow initial learning but flexible use of distractor configurations. Visual Cognition, 27(5-8), 563-575. doi.org/10.1080/13506285.2019.1668516

Salthouse, T. A. (1996). The processing-speed theory of adult age differences in cognition. Psychological Review, 103(3), 403-428. doi.org/10.1037/0033-295x.103.3.403

Shaw, R. J., \& Craik, F. I. M. (1989). Age differences in predictions and performance on a cued recall task. Psychology and Aging, 4(2), 131-135. doi.org/10.1037/0882-7974.4.2.131

Shih, S. I., Meadmore, K. L., \& Liversedge, S. P. (2012). Aging, eye movements, and objectlocation memory. PLoS One, 7(3), e33485. doi.org/10.1371/journal.pone.0033485

Smith, A. D., Park, D. C., Earles, J. L. K., Shaw, R. J., \& Whiting, W. L. (1998). Age differences in context integration in memory. Psychology and Aging, 13(1), 21-28. doi.org/10.1037/0882-7974.13.1.21

Smyth, A. C., \& Shanks, D. R. (2011). Aging and implicit learning: Explorations in contextual cuing. Psychology and Aging, 26(1), 127-132. doi.org/10.1037/a0022014

Spotorno, S., Malcolm, G. L., \& Tatler, B. W. (2015). Disentangling the effects of spatial inconsistency of targets and distractors when searching in realistic scenes. Journal of Vision, 15(2), 1-21. doi.org/10.1167/15.2.12

Taconnat, L., Raz, N., Toczé, C., Bouazzaoui, B., Sauzéon, H., Fay, S., \& Isingrini, M. (2009). Ageing and organisation strategies in free recall: The role of cognitive flexibility. European Journal of Cognitive Psychology, 21(2-3), 347-365. doi.org/10.1080/09541440802296413 
Tatler, B. W., Gilchrist, I. D., \& Land, M. F. (2005). Visual memory for objects in natural scenes: From fixations to object files. The Quarterly Journal of Experimental Psychology Section A, 58(5), 931-960. doi.org/10.1080/02724980443000430

Võ, M. L.-H., \& Henderson, J. M. (2009). Does gravity matter? Effects of semantic and syntactic inconsistencies on the allocation of attention during scene perception. Journal of Vision, 9(3), 24. doi.org/10.1167/9.3.24

Võ, M. L.-H., \& Wolfe, J. M. (2012). When does repeated search in scenes involve memory? Looking at versus looking for objects in scenes. Journal of Experimental Psychology: Human Perception and Performance, 38(1), 23-41. doi.org/10.1037/a0024147

Warren, D. E., Thurtell, M. J., Carroll, J. N., \& Wall, M. (2013). Perimetric evaluation of saccadic latency, saccadic accuracy, and visual threshold for peripheral visual stimuli in young compared with older adults. Investigative Opthalmology \& Visual Science, 54(8), 5778-5787. doi.org/10.1167/iovs.13-12032

Whiting, W. L., Madden, D. J., Pierce, T. W., \& Allen, P. A. (2005). Searching from the top down: ageing and attentional guidance during singleton detection. Quarterly Journal of Experimental Psychology, 58A(1), 72-97. doi.org/10.1080/02724980443000205

Wickham, H. (2009). ggplot2: Elegant graphics for data analysis. New York, NY: Springer.

Wiegand, I., \& Wolfe, J. M. (2020). Age doesn't matter much: Hybrid visual and memory search is preserved in older adults. Aging, Neuropsychology, and Cognition, 27(2), 220-253. doi.org/10.1080/13825585.2019.1604941

Williams, C. C. (2010). Not all visual memories are created equal. Visual Cognition, 18(2), 201228. doi.org/10.1080/13506280802664482

Williams, C. C., Henderson, J. M., \& Zacks, F. (2005). Incidental visual memory for targets and distractors in visual search. Perception \& Psychophysics, 67(5), 816-827. doi.org/10.3758/BF03193535 
Williams, C. C., Zacks, R. T., \& Henderson, J. M. (2009). Age differences in what is viewed and remembered in complex conjunction search. Quarterly Journal of Experimental Psychology, 62(5), 946-966. doi.org/10.1080/17470210802321976

Wolfe, J. M., Alvarez, G. A., Rosenholtz, R., Kuzmova, Y. I., \& Sherman, A. M. (2011). Visual search for arbitrary objects in real scenes. Attention, Perception, \& Psychophysics, 73(6), 1650-1671. doi.org/10.3758/s13414-011-0153-3

Woodbridge, R., Sullivan, M. P., Harding, E., Crutch, S., Gilhooly, K. J., Gilhooly, M. L. M., McIntyre, A., \& Wilson, L. (2018). Use of the physical environment to support everyday activities for people with dementia: A systematic review. Dementia, 17(5), 533-572. doi.org/10.1177/1471301216648670 


\section{Figure Captions}

Figure 1. Example of table layout: Two semantic groups of objects are highlighted according to the position conditions. The object of the first group, ID card, bank card, wallet and coin (outlined by a dotted circle), are placed near each other, while the objects of the other group, a tooth brushing set (outlined by circles), are placed far apart from each other. See the online version of the paper for a colored version of this figure.

Figure 2. Example of search table. The four searched targets (labeled S1/S2/S3/S4 according to the order in which they were looked for) are outlined considering the two position conditions (near vs. far from semantically associated objects). See the online version of the paper for a colored version of this figure.

Figure 3. Left panel: 1A) Search time in ms, as a function of age group and target position (near vs. far) with respect to the semantically associated objects. 1B) Fitted values, representing the effect on the scale of the linear predictors, as a function of age group and target position. Right panel: 2A) Search time, as a function of age group and incidental fixation (presence of fixations vs. no fixation) on the target when it was a distractor. 2B) Fitted values, as a function of age group and incidental fixation; this plot shows that the interaction between age group and incidental fixation was not significant. ${ }^{* * *}: p<.001,{ }^{*}: p$ $<.05$ and $n s:$ not significant. Error bars indicate \pm 1 standard error of the mean (SEM). See the online version of the paper for a colored version of this figure. Note that data from individual participants are provided in the online Supplementary Materials. 
Figure 1. Example of table layout: Two semantic groups of objects are highlighted according to the position conditions. The object of the first group, ID card, bank card, wallet and coin (outlined by a dotted circle), are placed near each other, while the objects of the other group, a tooth brushing set (outlined by circles), are placed far apart from each other. See the online version of the paper for a colored version of this figure.

$198 \times 114 \mathrm{~mm}$ ( $300 \times 300$ DPI) 
Figure 2. Example of search table. The four searched targets (labeled S1/S2/S3/S4 according to the order in which they were looked for) are outlined considering the two position conditions (near vs. far from semantically associated objects). See the online version of the paper for a colored version of this figure.

$$
204 \times 107 \mathrm{~mm}(300 \times 300 \text { DPI })
$$



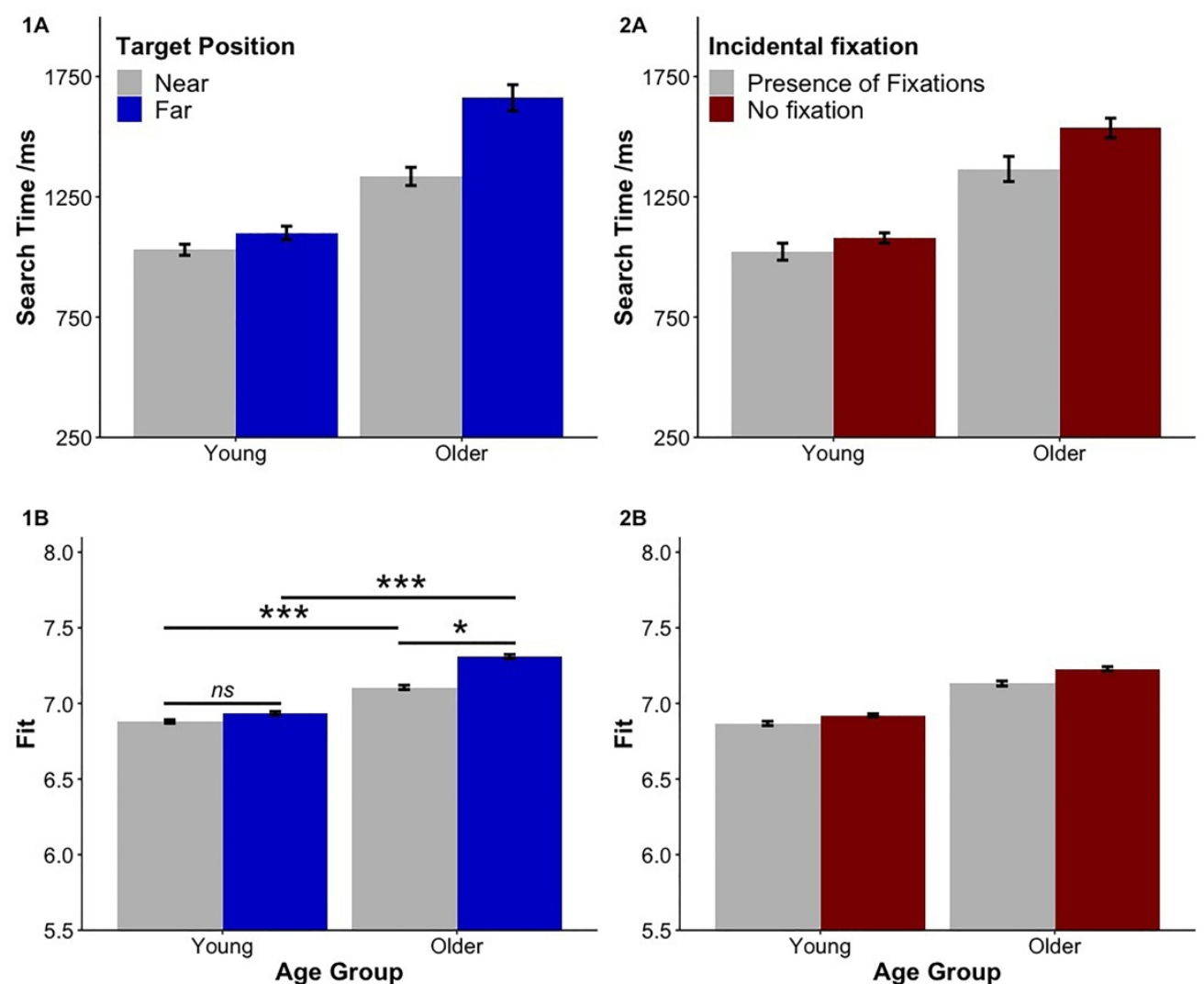

Figure 3. Left panel: 1A) Search time in ms, as a function of age group and target position (near vs. far) with respect to the semantically associated objects. 1B) Fitted values, representing the effect on the scale of the linear predictors, as a function of age group and target position. Right panel: $2 \mathrm{~A}$ ) Search time, as a function of age group and incidental fixation (presence of fixations vs. no fixation) on the target when it was a distractor. 2B) Fitted values, as a function of age group and incidental fixation; this plot shows that the interaction between age group and incidental fixation was not significant. $* * *: p<.001, *: p<.05$ and ns: not significant. Error bars indicate \pm 1 standard error of the mean (SEM). See the online version of the paper for a colored version of this figure. Note that data from individual participants are provided in the online Supplementary Materials.

\section{$363 \times 299 \mathrm{~mm}(300 \times 300 \mathrm{DPI})$}

